\title{
latrogenic common carotid artery rupture during neck surgery rescued using covered stent - A case report -
}

Received August 10, 2017

Revised 1st, October 17, 2017

2nd, December 15, 2017

3rd, December 22, 2017

4th, December 28, 2017

Accepted December 29, 2017

\section{Corresponding author}

Il Woo Shin, M.D., Ph.D.

Department of Anesthesiology and

Pain Medicine, Gyeongsang National

University Hospital, Gyeongsang

National University School of

Medicine, 79 Gangnam-ro, Jinju

52727, Korea

Tel: 82-55-750-8141

Fax: 82-55-750-8142

E-mail: ilwooshin@gnu.ac.kr

\section{ORCID}

http://orcid.org/0000-0003-1964-4546

\section{Ji Yoon Kim ${ }^{1}$, Il Woo Shin ${ }^{1,2,3}$, Sunmin Kim', Se-bin Kang1, Soo-hee Lee ${ }^{1,2}$, Kyeong Eon Park', Heon Keun Lee ${ }^{1,2}$, Ju-Tae Sohn ${ }^{1,2,3}$, and Young Kyun Chung ${ }^{1,2}$}

Department of Anesthesiology and Pain Medicine, 'Gyeongsang National University

Hospital, ${ }^{2}$ Gyeongsang National University School of Medicine, ${ }^{3}$ Institute of Health Sciences, Gyeongsang National University, Jinju, Korea

\begin{abstract}
Carotid artery rupture during head and neck surgery is a catastrophic, life-threatening emergency. Although recent incidence has declined, it still occurs in many patients. Hemorrhage from the carotid artery is usually massive and uncontrollable. Fast, aggressive treatment to prevent hemodynamic instability is required. Even if patients survive this event, they may experience severe neurological sequelae. A ruptured carotid artery is usually controlled by direct compression and arterial ligation. However, apart from the inherent difficulty of operation, these traditional surgical treatments are associated with high morbidity and mortality. In the past two decades, endovascular management has become a mainstay of carotid rupture treatment. We report a case of successful recovery without any sequelae after cardiovascular collapse due to an unintentional common carotid artery (CCA) rupture during neck surgery. The exposed CCA was treated with a covered stent. In such a case, multidisciplinary cooperation is crucial.
\end{abstract}

Key Words: Anesthesia, Common carotid artery, Interventional radiology, Stents.
The brain, which is highly vulnerable to ischemia, receives its blood supply from the internal carotid arteries (ICAs) and vertebral arteries. Within the cranial vault, the terminal branches of these arteries form an anastomotic circle, called the Circle of Willis. However, hypoplasia of these circles is common (24\%), especially in the posterior communicating artery, which was the most frequent artery found to be hypoplastic [1]. The ICA is derived from the common carotid artery (CCA). Therefore, ICA or CCA rupture may cause a significant decrease in cerebral blood flow and lead to the most devastating complications, such as cerebral ischemia and even death.

There are several etiologies of carotid blowout. Risk factors associated with carotid blowout include wound breakdown, necrosis, infection, pharyngocutaneous fistula, prior radiation therapy, tumor involvement of the arterial wall, and aggressive handling of the adventitia during dissection [2]. Carotid artery rupture is not a common complication during neck surgery, but it does occur in several patients.

We present a case of successful rescue after iatrogenic CCA rupture during metastatic lymph node dissection of thyroid papillary carcinoma. After moving the patient from the operating room to the interventional radiologic suite, the exposed artery was repaired using a covered stent.

\section{CASE REPORT}

A 31-year-old man who was $171 \mathrm{~cm}$ tall and weighed $85 \mathrm{~kg}$

This is an Open Access article distributed under the terms of the Creative Commons Attribution Non-Commercial License (http://creativecommons.org/licenses/by-nc/4.0) which permits unrestricted non-commercial use, distribution, and reproduction in any medium, provided the original work is properly cited. 
was scheduled for lymph node excision under general anesthesia. He was diagnosed with thyroid cancer 4 years prior, and underwent a total thyroidectomy with modified radical lymph node dissection of the neck. He was taking levothyroxine sodium for replacement of thyroid hormone. During outpatient treatment after surgery, a 3.3-cm sized cystic lesion was found in the right supraclavicular area. To rule out metastatic lymph node disease, lymph node excision was planned under general anesthesia.

After sufficient preoxygenation, general anesthesia was induced with intravenous administration of sodium thiopental (400 mg). The patient was manually ventilated with $100 \%$ oxygen and received intravenous administration of rocuronium (60 mg) for muscle relaxation. Orotracheal intubation was performed with a reinforced endotracheal tube (internal diameter of $7.5 \mathrm{~mm}$ ). Anesthesia was maintained with oxygen delivery at a rate of $1.5 \mathrm{~L} / \mathrm{min}$, nitrous oxide at $1.5 \mathrm{~L} / \mathrm{min}$, and sevoflurane, 2.0 volume\%.

After dissection, metastatic lymph node invasion of the right CCA was confirmed. During careful dissection, the CCA ruptured unexpectedly and massive arterial spurting occurred. For bleeding control, the surgeon applied direct manual compression with the hand. During compression, anesthesiologists placed an arterial line in the left dorsalis pedis artery, a central line in the right femoral vein, and an additional peripheral venous line in the left foot (18 gauge). While waiting for blood product preparation, $1,000 \mathrm{ml}$ of colloid (Volulyte ${ }^{\circledR}$, Balanced 6\% hydroxyethyl 130/0.4, Frenius Kabi, Germany) was infused for resuscitation. To provide protection from brain damage, methylprednisolone (500 mg), ulinastatin (Ulistin ${ }^{\circledast}, 100,000 \mathrm{IU} / 2 \mathrm{ml}$, Han Lim Pharm. Co. Ltd., Korea) (200,000 IU), and sodium thiopental (250 $\mathrm{mg}$ ) were administered intravenously.

Several attempts to directly repair the ruptured CCA failed, so vascular surgeons were called for assistance. Vascular surgeons, otolaryngologists, and anesthesiologists discussed the clinical situation. An attempt at direct repair of the CCA using hypothermic cardiopulmonary bypass and total circulatory arrest was considered for a moment, but then it was concluded that endovascular management was a better choice to ensure the patient's safety. After talking with the interventional radiologists, the anesthesiologists and surgeons moved the patient to the interventional radiology suite about 300 meters from the operating room while compressing the ruptured
CCA. During the 5-minute shift, the patient was ventilated using a manually operated ventilation bag $\left(\mathrm{Ambu}^{\circledR}\right.$ Mark IV Resuscitators, AMBU, Denmark) via endotracheal tube, received a blood transfusion, and was administered sodium thiopental (500 mg) by continuous intravenous infusion. Upon arrival in the interventional radiology suite, the patient was connected to a prepared anesthetic machine.

The procedure was approached via the right femoral artery. Extravasation of the contrast agent from the right proximal CCA occurred within the neck (Fig. 1). Along the guide wire, the covered stent (Fig. 2) was inserted into the right proximal CCA (Fig. 3). There was massive bleeding during intermittent release of CCA compression, which was done to check the results of the procedure. During the procedure, the patient continued to receive blood transfusion and norepinephrine at $0.1 \mathrm{mcg} / \mathrm{kg} / \mathrm{min}$ by continuous intravenous infusion.

The angiographic findings showed complete closure of the rupture site. The distal flow to the CCA and subclavian artery was well maintained, and the procedure was terminated. The patient was transferred back to the operating room. After arriving in the operating room, a lymph node biopsy and wound closure were performed. Finally, the surgery was completed.

Durations of anesthesia, endovascular procedure, and surgery were 4 hours and 40 minutes, 1 hour, and 2 hours,

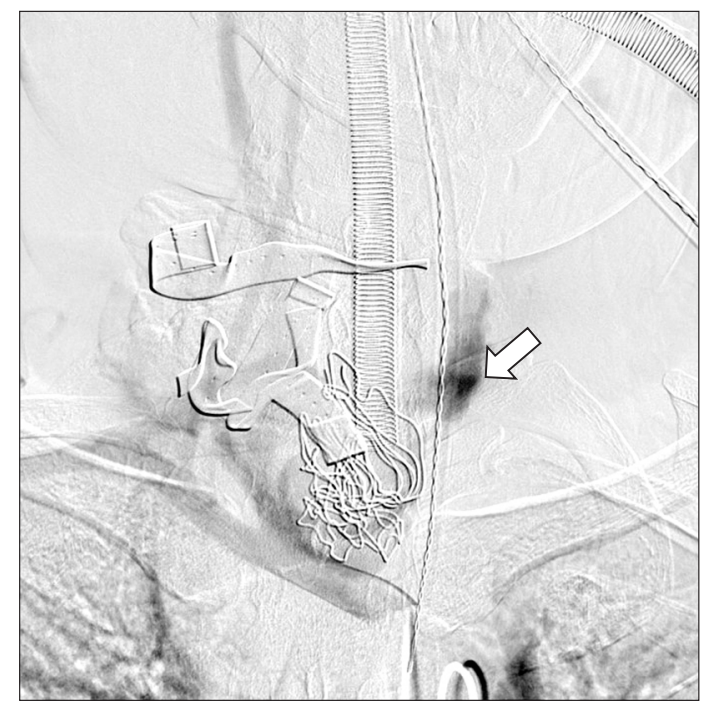

Fig. 1. Angiography of a ruptured common carotid artery. The site of rupture is compressed by gauze packing and a hand. This carotid angiography shows a bleeding focus of extravasation and decreased blood flow of the internal and external carotid arteries. 


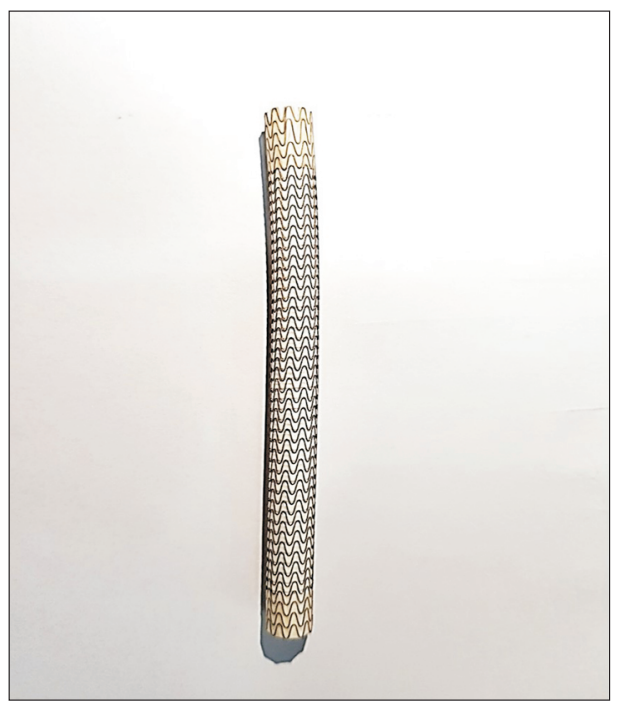

Fig. 2. Covered stent. A covered stent is composed of a special fabric and metal (W. L. Gore and Associates, USA).

respectively. During this time, vital signs were maintained at the following parameters: systolic blood pressure, 78-163 mmHg; diastolic blood pressure, 41-97 mmHg; heart rate, $80-111$ beats $/ \mathrm{min}$; body temperature $35.0^{\circ} \mathrm{C}-35.7^{\circ} \mathrm{C}$; pulse oximetry, $98 \%-100 \%$. Mechanical ventilation was secured at constant settings that included a tidal volume of $550-600 \mathrm{ml}$, a respiratory rate of 10-14 breaths/min, end-tidal carbon dioxide of 30-36 mmHg, and peak inspiratory pressure of 20-22 $\mathrm{cmH}_{2} \mathrm{O}$. The bispectral index was maintained at 22-47. Additionally, the patient was infused with crystalloid (3,100 ml), colloid (1,000 ml), and transfused with an additional 8 units of packed red blood cells $(2,000 \mathrm{ml}), 6$ units of fresh frozen plasma (1,500 ml), and 10 units of platelet concentrate $(1,500$ $\mathrm{ml})$. His urine output was $670 \mathrm{ml}(143 \mathrm{ml} / \mathrm{h})$, and the estimated blood loss was 4,300 $\mathrm{ml}$. Immediately after the operation, the hemoglobin level was $14.2 \mathrm{~g} / \mathrm{dl}$, the hematocrit was $43 \%$, and the platelet count was $205,000 / \mathrm{mm}^{3}$.

The patient was transferred to the surgical intensive care unit (SICU) with norepinephrine administered at $0.05 \mu \mathrm{g} / \mathrm{kg} /$ min via continuous infusion. The patient's vital signs at the SICU were stable. No specific findings were observed during the postoperative brain work-up; therefore, the patient was extubated later that same day. He was shifted to the medical ward one day later and discharged eight days after the surgery.

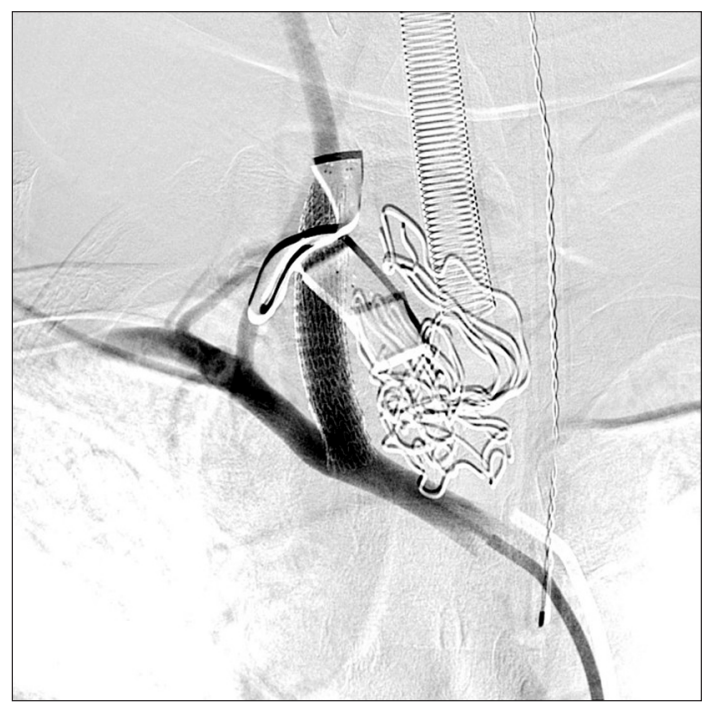

Fig. 3. Ruptured common carotid artery successfully repaired by stent. This carotid angiography demonstrates well-maintained distal flow to the carotid artery and subclavian artery.

\section{DISCUSSION}

In most areas of the world, the prevalence rate of thyroid cancer has rapidly increased compared to the past due to the increased use of advanced imaging techniques [3]. Most patients who present with thyroid carcinoma have well-differentiated histology and have an excellent prognosis. However, lymph node metastasis is relatively common in patients with papillary thyroid cancer ( $30 \%-80 \%)$, and nodal metastasis is a source of recurrent disease [4], as in this present case.

Thyroid surgery is considered a relatively safe procedure (mortality rate: $0.065 \%$ ) [5]. However, various complications are associated with neck dissections. More specifically, with central neck dissection, hypoparathyroidism, recurrent laryngeal nerve palsy, external laryngeal nerve injury, or injury to the trachea or esophagus can occur. Complications that can occur with lateral neck dissection include injury to the following structures: the thoracic duct, the internal jugular vein, the carotid artery, the spinal accessory nerve, superficial cervical nerves, the hypoglossal nerve, the brachial plexus, the phrenic nerve, and the sympathetic cervical plexus. Other complications include pneumothorax, seroma, wound infection, and hemorrhage [6]. However, among these complications, carotid artery rupture may be the most feared complication of neck surgery $[2,7]$.

The reported incidence of carotid artery rupture in patients 
who have undergone neck dissection is approximately $3 \%-4 \%$ in older reports, but the current incidence has been reduced to $0 \%-1.2 \%$ because of advances in the management of these patients [2]. Even under the best circumstances, the mortality is unacceptably high. Even if a patient survives, the sequelae resulting from cerebral ischemia can be devastating. Although there are differences in each case, reported neurologic morbidity is $40 \%$ and mortality is $25 \%$ [2].

There are several ways to deal with intraoperative injuries to the CCA, including repair using primary sutures. Another type of surgical management is vessel ligation and/or bypass. However, there is a technical difficulty of dealing with anatomy altered by prior surgery and radiation, resulting in tissues that are fragile and poorly healed. Aggressive handling of blood vessels causes more tearing of the adventitia of the vessel; therefore, it can make the situation significantly worse. These methods are not only surgically difficult but are also associated with a higher neurologic morbidity rate (50\%) than endovascular management mentioned in the following paragraphs [2,7].

The first description of the use of transcatheter embolization in the internal iliac artery to control hemorrhage associated with pelvic fractures was published in 1972. Since then, the role of interventional radiologists in trauma has evolved from that of making the initial diagnosis of vascular and solid organ injuries to temporizing or providing definitive treatment [8]. Additionally, after the mid-1990s, endovascular techniques have become mainstay treatment options for carotid artery rupture [7]. Endovascular management can be deconstructive, in which the vessel is sacrificed, or reconstructive, in which the vessel is left patent by the placement of covered stents.

Balloon occlusion and coil embolization are sacrificing procedures. However, as many as $15 \%-20 \%$ of patients who experience carotid blowouts that are managed with deconstructive procedures may develop immediate or delayed cerebral ischemia due to an incomplete circle of Willis, thromboembolism arising from an acutely occluded carotid artery, or delayed collateral failure [9]. Some authors have advocated percutaneous balloon test occlusion (BTO) prior to definitively sacrificing the vessel. However, BTO is often bypassed inevitably due to the emergency of the clinical situation. Even if BTO is performed, it does not completely prevent neurologic complications. Covered stent placement is a reconstructive procedure that maintains blood flow to the cerebral circulation. Some investigators have proposed the use of covered stents in patients who fail BTO or are perceived as having limited collateral flow based on an incomplete circle of Willis. However, endovascular management is still associated with complications such as stent occlusion, stent infection, and brain abscess due to the placement of foreign bodies, which reduces morbidity and mortality $[2,7]$.

Direct repair of the CCA using cardiopulmonary bypass was also considered for a while, based on earlier reports. Cardiopulmonary bypass, an indispensable tool for cardiac surgery, can also be used successfully in patients who require induced hypothermia and total circulatory arrest to extract a large thrombus or tumors that are highly vascular, helping to reduce fatal hemorrhage. Furthermore, femoro-femoral cardiopulmonary bypass would have been a suitable alternative for the institution of cardiopulmonary bypass via a median sternotomy. However, cardiopulmonary bypass has many well-known complications [10]. Therefore, endovascular management was chosen to enhance the patient's safety, instead of cardiopulmonary bypass.

After an acute carotid blowout during neck surgery, a patient presents with acute vigorous hemorrhage that is neither self-limited nor well controlled by packing, and the patient deteriorates rapidly. Therefore, immediate aggressive intervention of anesthesiologists is necessary. Securing the patient's airway is an obvious necessity. Fluid resuscitation should be initiated. At least two and preferably four largebore intravenous lines should be secured. Compression of the bleeding site should be applied by the surgeon. Intensive monitoring, including central venous access and an arterial line, is required. Vascular surgery consultation is highly recommended. After endovascular management or operative ligation, the patient should be monitored in an intensive care unit for signs of cerebral ischemia. Additionally, clinical information including blood pressure, heart rate, intravascular volume, and hematocrit should be optimized with careful monitoring [2].

In addition to these efforts, there are several strategies to reduce cerebral injury. It was reported that barbiturate, propofol, inhalation anesthetics (isoflurane, servoflurane, and desflurane), moderate hypothermia, hyperbaric oxygen, lidocaine, and ketamine provide cerebral protection in the experimental model involving cerebral ischemia [11]. Thiopen- 
tal, a barbiturate, is used to achieve electroencephalographic suppression and maximally reduce cerebral metabolism. In addition, methylprednisolone, a potent anti-inflammatory corticosteroid is of great value in anti-apoptosis of the central nervous system. According to some surveys regarding medications used for cerebral protection in deep hypothermia circulatory arrest, the median dose of sodium thiopental and methylprednisolone was 250 to 3,000 and 500 to $2,250 \mathrm{mg}$, respectively, suggesting that the dosage is variable. Although the magnitude of cerebral ischemia induced by carotid artery rupture may be different from that of cerebral ischemia induced by deep hypothermia circulatory arrest, the dosage of thiopental (250 mg) and methylprednisolone (500 mg) used in this patient was within the range of dosage induced by other clinicians for cerebral protection [12]. Ulinastatin (a urinary trypsin inhibitor) is known to play an important role in the protection of organs including brain against ischemic injury during severe inflammation. According to an animal study, it plays a beneficial role in modulating global cerebral ischemia-induced cardiac arrest. Although it was reported that ulinastatin is effective for brain protection in the global ischemia and acute hydyrogen sulfide intoxicated rats, to our best knowledge, the effect of ulinastatin regarding brain protection in human is not available. Therefore, further study to regrade the effect of ulinasatin on cerebral protection in the human is needed $[13,14]$. In summary, despite decades of work, the evidence of benefit of these current strategies remain sparse, and no strategies have yet been approved as specific neuroprotective strategies. The search for such strategies should be continued.

An additional point that cannot be left unsaid concerns the presence of carotid sinus baroreceptor (CSB) at the bifurcation of the CCA. The CSB is a stretch-sensitive mechanoreceptor that responds to alterations in blood pressure. The CSBs are located within the adventitia of the origin of the ICA and are innervated by the carotid sinus nerve, which is a branch of the glossopharyngeal nerve. Operative stimulation such as stretching can result in perioperative hemodynamic instability. Likewise, during carotid stenting, balloon inflation performed to expand the carotid stent may lead to excessive stimulation of the carotid baroreceptor, resulting in hemodynamic instability [15]. Fortunately, there were no such events in this case. However, it requires the anesthesiologists' attention.
In this case, the rupture was not anticipated, despite various risk factors, including the previous neck dissection history and vessel involvement of cancer. However, multidisciplinary teams led by anesthesiologists responded rapidly to a hemodynamic crisis. Therefore, the patient could undergo surgery without any complication. Clinicians need to be mindful of the importance of a team-oriented collaboration.

This case suggests that when major vascular damage occurs, interventional radiology techniques can serve as treatment. As the field of interventional radiology is rapidly and continuously evolving, anesthesiologists must be aware that the use of interventional radiology is an option when severe major vessel or organ injury occurs in the perioperative period. This has enabled us to reduce the risk of life-threatening hemorrhage and a devastating complication, such as cerebral infarct. Furthermore, it is important to determine the emergency intervention before cardiovascular collapse. Lastly, hybrid operating rooms that contain both surgical equipment and vascular imaging equipment have been in the spotlight in recent years. If the hospital has these facilities, planning this type of surgery in a hybrid operating room seems to be ideal.

\section{REFERENCES}

1. Iqbal S. A comprehensive study of the anatomical variations of the circle of willis in adult human brains. J Clin Diagn Res 2013; 7: 2423-7.

2. Citardi MJ, Chaloupka JC, Son YH, Ariyan S, Sasaki CT. Management of carotid artery rupture by monitored endovascular therapeutic occlusion (1988-1994). Laryngoscope 1995; 105: 1086-92.

3. La Vecchia C, Malvezzi M, Bosetti C, Garavello W, Bertuccio P, Levi F, et al. Thyroid cancer mortality and incidence: a global overview. Int J Cancer 2015; 136: 2187-95.

4. Hamming JF, Roukema JA. Management of regional lymph nodes in papillary, follicular, and medullary thyroid cancer. In: Textbook of endocrine surgery. 3rd ed. Edited by Clark OH, Duh QY, Kebebew E, Gosnell JE, Shen WT: New Delhi, Jaypee Brothers Medical Publishers Ltd. 2016, pp 407-8.

5. Gómez-Ramírez J, Sitges-Serra A, Moreno-Llorente P, Zambudio AR, Ortega-Serrano J, Rodríguez MT, et al. Mortality after thyroid surgery, insignificant or still an issue? Langenbecks Arch Surg 2015; 400: 517-22.

6. Cheah WK, Arici C, Ituarte PH, Siperstein AE, Duh QY, Clark OH. Complications of neck dissection for thyroid cancer. World J Surg 
2002; 26: 1013-6.

7. Haas RA, Ahn SH. Interventional management of head and neck emergencies: carotid blowout. Semin Intervent Radiol 2013; 30: 245-8.

8. Zealley IA, Chakraverty S. The role of interventional radiology in trauma. BMJ 2010; 340: c497.

9. Lesley WS, Chaloupka JC, Weigele JB, Mangla S, Dogar MA. Preliminary experience with endovascular reconstruction for the management of carotid blowout syndrome. AJNR Am J Neuroradiol 2003; 24: 975-81.

10. Song JH, Lee HS, Han JU, Jung JK, Kim HZ, Shinn HK. Anesthetic management for liver segmentectomy and thrombectomy of inferior vena cava and right atrium without cardiopulmonary bypass: a case report. Anesth Pain Med 2007; 2: 224-7.

11. Fukuda S, Warner DS. Cerebral protection. Br J Anaesth 2007; 99:
10-7.

12. Dewhurst AT, Moore SJ, Liban JB. Pharmacological agents as cerebral protectants during deep hypothermic circulatory arrest in adult thoracic aortic surgery. A survey of current practice. Anaesthesia 2002; 57: 1016-21.

13. Zhou J, Lian J, Li HX, Hong GL, Zhao GJ, Zhi SC, et al. [Mechanism research and effect of ulinastatin in the brain tissue injury of acute hydrogen sulfide intoxicated rats]. Chin J Ind Hyg Occup Dis 2016; 34: 166-72.

14. Jiang XM, Hu JH, Wang LL, Ma C, Wang X, Liu XL. Effects of ulinastatin on global ischemia via brain pro-inflammation signal. Transl Neurosci 2016; 7: 158-63.

15. Demirci M, Saribaş O, Uluç K, Cekirge S, Böke E, Ay H. Carotid artery stenting and endarterectomy have different effects on heart rate variability. J Neurol Sci 2006; 241: 45-51. 